\title{
๖Nonlinear Generation of Long Waves and the Reversal of Eddy Momentum Fluxes in a Two-Layer Quasigeostrophic Model
}

\author{
Tsung-Lin Hsieh, ${ }^{\mathrm{a}}$ Chiung-Yin Chang, ${ }^{\mathrm{b}}$ IsaAc M. Held,${ }^{\mathrm{a}}$ And Pablo Zurita-Gotor ${ }^{\mathrm{c}}$ \\ ${ }^{a}$ Program in Atmospheric and Oceanic Sciences, Princeton University, Princeton, New Jersey \\ ${ }^{\mathrm{b}}$ Department of the Geophysical Sciences, University of Chicago, Chicago, Illinois \\ ${ }^{\mathrm{c}}$ Instituto de Geociencia, Centro Mixto del Consejo Superior de Investigaciones Científicas, \\ Universidad Complutense de Madrid, Madrid, Spain
}

(Manuscript received 4 December 2020, in final form 11 July 2021)

\begin{abstract}
Although classical theories of midlatitude momentum fluxes focus on the wave-mean flow interaction, wave-wave interactions may be important for generating long waves. It is shown in this study that this nonlinear generation has implications for eddy momentum fluxes in some regimes. Using a two-layer quasigeostrophic model of a baroclinic jet on a $\beta$ plane, statistically steady states are explored in which the vertically integrated eddy momentum flux is divergent at the center of the jet, rather than convergent as in Earthlike climates. One moves toward this less familiar climate from more Earthlike settings by reducing either $\beta$, frictional drag, or the width of the baroclinic zone, or by increasing the upper bound of resolvable wavelengths by lengthening the zonal channel. Even in Earthlike settings, long waves diverge momentum from the jet, but they are too weak to compete with short unstable waves that converge momentum. We argue that long waves are generated by breaking of short unstable waves near their critical latitudes, where long waves converge momentum while diverging momentum at the center of the jet. Quasi-linear models with no wave-wave interaction can qualitatively capture the Earthlike regime but not the regime with momentum flux divergence at the center of the jet, because the nonlinear wave breaking and long-wave generation processes are missing. Therefore, a more comprehensive theory of atmospheric eddy momentum fluxes should take into account the nonlinear dynamics of long waves.
\end{abstract}

KEYWORDS: Momentum; Rossby waves; Quasigeostrophic models

\section{Introduction}

The convergence of eddy momentum flux toward the jet in the midlatitude atmosphere is one of the most fundamental questions in the study of the general circulation. The classical explanation relies on the midlatitudes being the source of eddies that then propagate or migrate poleward and especially equatorward, so that the dissipation of the eddies is displaced in latitude from their source. From the general perspective of pseudomomentum (i.e., wave activity) conservation, the momentum flux convergence is determined by the difference between the source and sink of pseudomomentum. See Vallis (2017) for an exposition of this classical framework.

Since baroclinic instability is the eddy source, surface westerlies are expected in the region of maximal instability. In the simplest quasi-linear picture, the eddy momentum fluxes are associated with meridionally propagating Rossby waves, with the direction of the eddy momentum flux opposite to the direction of the group velocity, and with the sink determined by the location of wave breaking and dissipation as these waves approach their critical latitudes in the upper troposphere. See Randel and Held (1991) for observational support for this quasi-linear perspective on the eddy momentum flux.

¿ Denotes content that is immediately available upon publication as open access.

Corresponding author: Tsung-Lin Hsieh, hsiehtl@princeton.edu
The picture of eddy momentum fluxes as determined by the characteristics of the decay phase of baroclinically generated eddies as opposed to the growth stage, discussed originally by Kuo (1951), is clearly demonstrated in the simulations of the nonlinear life cycles of unstable baroclinic waves by Simmons and Hoskins (1978). This decay phase can be controlled by mechanisms differing from those controlling the heat fluxes that are more tightly connected to the linear growth stage, making the development of theories for the momentum fluxes particularly challenging.

Despite its success in qualitatively explaining Earth's mean climate, spectral analyses of the observations (Lutsko et al. 2017) and idealized global models (O'Rourke and Vallis 2016) suggest that sufficiently long, planetary-scale waves do not follow this classical, quasi-linear paradigm. These waves diverge momentum from the jet, but this divergence typically exists in the lower troposphere and the flux amplitudes are relatively insignificant. The work of Lee (1997) on the transition from one storm track and its associated eddy-driven jet to two storm tracks also points to the potential importance of eddy momentum fluxes in baroclinic systems that transport momentum out of jets. Yet the mechanisms for the generation of the long, momentum-diverging waves were unclear.

In this study, we explore the parameter dependence of such momentum-diverging waves in a two-layer quasigeostrophic (QG) model of a baroclinically unstable jet on a $\beta$ plane and show the conditions in which they can become dominant. This model is particularly suitable for our purpose because it allows explicit control of $\beta$ independent of the Coriolis parameter $f$ (unlike spherical models), and it is the simplest model that 
encapsulates baroclinic instability and Rossby wave propagation. It is also encouraging that the two-layer model generates baroclinic life cycles that are qualitatively very similar to those in a multilayer model studied by Simmons and Hoskins (1978), as long as the linearly unstable mode that provides the initial condition has an upper-tropospheric critical latitude, as demonstrated by Feldstein and Held (1989).

The two-layer QG model has been an important test bed for studies of eddy fluxes in statistically steady states since the pioneering work of Phillips (1956). As in all QG models, a theory for the eddy fluxes can be framed as a theory for the eddy potential vorticity (PV) fluxes. The sum of the two PV fluxes (which are typically of opposite sign in the two layers) provides the vertically integrated momentum flux convergence and the difference provides the eddy heat flux. But the momentum flux convergence is generally a small difference between the PV and heat fluxes, so theories that take the PV fluxes as a point of departure [e.g., the diffusive approach in Wiin-Nielsen and Sela (1971) and Pavan and Held (1996)], are not easily converted into adequate theories for the momentum flux. Theories that do exist rely on a quasi-linear, wave-mean flow interaction perspective, such as the stochastic theories of Farrell and Ioannou (2003). Therefore, a useful starting point for any attempt at a theory of the momentum fluxes in the twolayer model is to clarify the parameter regime in which a quasilinear approximation is adequate.

In this study, we address the basic question of the existence of a regime having momentum flux divergence at the latitude of maximal baroclinic instability, in which regime the longwave dynamics are important and the quasi-linear perspective is insufficient. The model is described in section 2. In section 3, examples are described of the two regimes, one with momentum flux convergence and another with momentum flux divergence at the center of the jet. A mechanism is proposed and tested in initial value experiments in section 4. Dependence of the momentum flux direction is explored in the larger parameter space in section 5 .

\section{A forced-dissipative two-layer QG model}

Our two-layer QG model is set up in a zonally periodic channel with thermal relaxation and surface drag. It is similar to that used in many other papers, such as Lee and Held (1993), Pavan and Held (1996), Zurita-Gotor and Lindzen (2006), and Lutsko et al. (2015). With the small scale diffusive terms not shown explicitly, the dimensional equations of motion are

$$
\begin{aligned}
& \frac{\partial q_{1}}{\partial t}=-J\left(\psi_{1}, q_{1}\right)-\beta v_{1}+r_{T} \frac{\tau-\tau_{E}}{\lambda^{2}} \\
& \frac{\partial q_{2}}{\partial t}=-J\left(\psi_{2}, q_{2}\right)-\beta v_{2}-r_{T} \frac{\tau-\tau_{E}}{\lambda^{2}}-r_{m} \nabla^{2} \psi_{2}
\end{aligned}
$$

where $q_{i}=\nabla^{2} \psi_{i}+(-1)^{i}\left(\psi_{1}-\psi_{2}\right) / \lambda^{2}$ is the perturbation QG potential vorticity from the background $\beta y, \psi_{i}$ is the geostrophic streamfunction, $\tau=\psi_{1}-\psi_{2}$ is the baroclinic streamfunction, $u_{i}=-\partial_{y} \psi_{i}$ and $v_{i}=\partial_{x} \psi_{i}, \lambda$ is the deformation radius, $r_{T}$ is the thermal relaxation rate, and $r_{m}$ is the mechanical damping rate. The index $i=1$ represents the upper layer and $i=2$ the lower layer. The mechanical drag occurs only in the lower layer. The layers have equal depth when at rest.

In this forced-dissipative system, baroclinicity is maintained by linearly relaxing $\tau$ to an equilibrium profile $\tau_{E}$. This equilibrium profile is purely a function of latitude $y$ and is antisymmetric across $y=0$, resulting in a symmetric jet:

$$
\tau_{E}(y)=\tau_{0} \tanh (y / \sigma)
$$

where $\sigma$ characterizes the meridional scale. The vertical wind shear in thermal wind balance with this profile at $y=0$ is denoted by $U=\tau_{0} / \sigma$. Hereafter, all variables are nondimensionalized by $U$ and $\lambda$.

The equilibrium profile $\tau_{E}$ is zonally uniform, so the thermal relaxation term simultaneously strengthens the zonal-mean jet and damps the eddy perturbation in $\tau$. The system is continuously forced by baroclinic instability and dissipated by the linear damping terms, and it eventually reaches a statistically steady state. A useful relationship in the statistically steady state is the balance between the vertically integrated convergence of eddy momentum flux (EMF) and surface drag:

$$
\sum_{i=1,2}\left(-\partial_{y} \overline{u_{i}^{\prime} v_{i}^{\prime}}\right)=r_{m} \bar{u}_{2} .
$$

That is, the direction of surface (lower layer) wind corresponds to the convergence or divergence of EMF at each latitude. The convergence of EMF is equivalent to the vorticity flux in either layer, and the zonal-mean PV flux is the combination of the vorticity flux and the heat flux:

$$
\begin{aligned}
& \overline{v_{i}^{\prime} \zeta_{i}^{\prime}}=-\partial_{y} \overline{u_{i}^{\prime} v_{i}^{\prime}}, \\
& \overline{v_{i}^{\prime} q_{i}^{\prime}}=\overline{v_{i}^{\prime} \zeta_{i}^{\prime}}+(-1)^{i} \overline{v_{i}^{\prime} \tau^{\prime}} .
\end{aligned}
$$

We utilize the numerical solver for this QG system developed by Abernathey et al. (2019) that has been used in studies of QG turbulence including Wang et al. (2016) and Zhang et al. (2020). The model is implemented in a doubly periodic square domain with an opposite $\tau_{E}$ added at $y=L / 2$, where $L=128$ is the channel length as well as domain width unless specified otherwise. That is, the jet of interest occurs at $y=0$, and a reversed jet occurs at $y=L / 2$ to be consistent with the periodic setup. To suppress eddy activity around the reversed jet, a local damping is applied in the vicinity of $y=L / 2$ with width $\Delta y=6$, but in practice the jets are far enough apart that the opposite jet, regardless of local damping, has little influence on the dynamics around $y=0$. The spectral resolution is 256 modes in the $x$ and $y$ directions with a fixed time step. Further increase of resolution makes negligible difference in the solution statistics. A spectral filter whose strength increases exponentially from $65 \%$ of the largest wavenumber is applied to prevent numerical instability (Abernathey et al. 2019).

\section{Opposite signs of EMF with different values of $\beta$}

In this section, we demonstrate the existence of a regime with EMF divergence at the center of the baroclinically unstable region, as the $\beta$ parameter is decreased toward zero. The anomalous EMF direction occurs in the upper layer as well as 
(a)

$\beta=0.2$

Upper layer potential vorticity

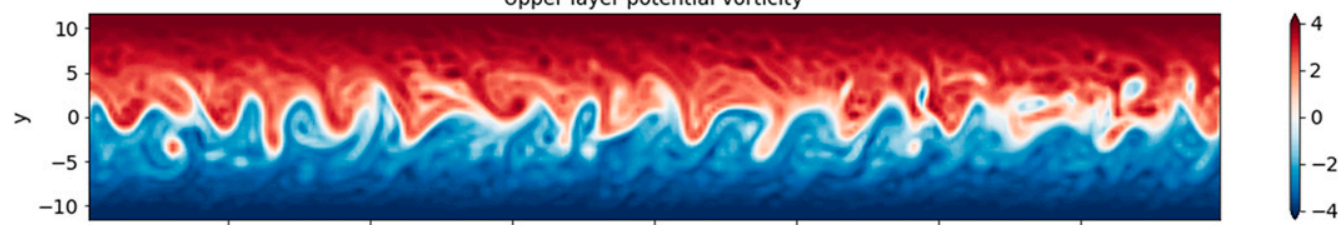

(b)

Lower layer potential vorticity

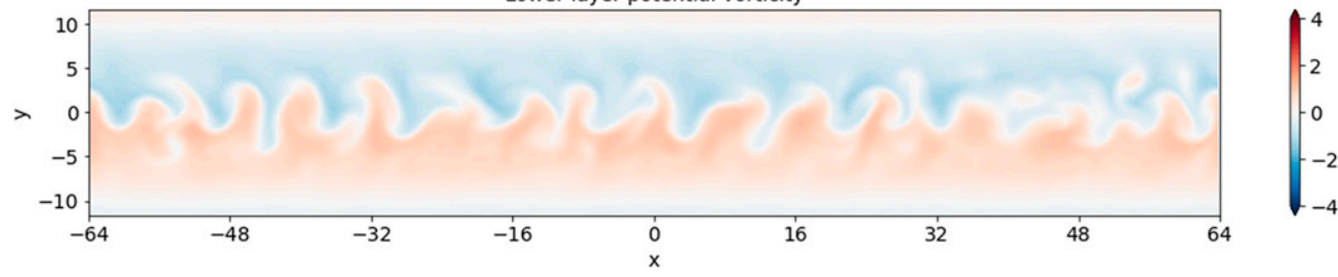

(c)

$\beta=0.02$

Upper layer potential vorticity

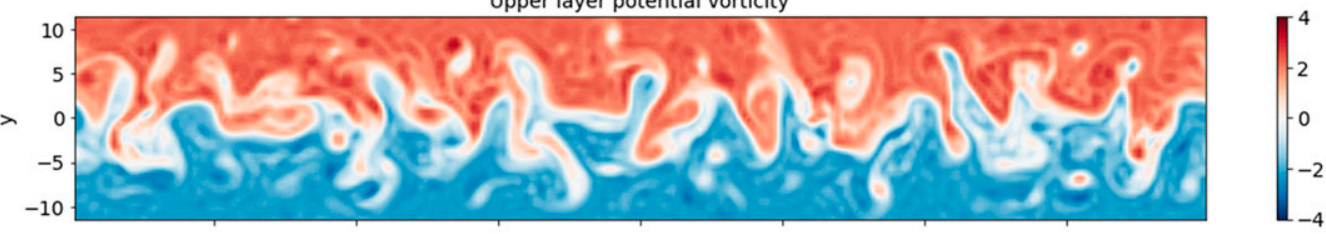

(d)

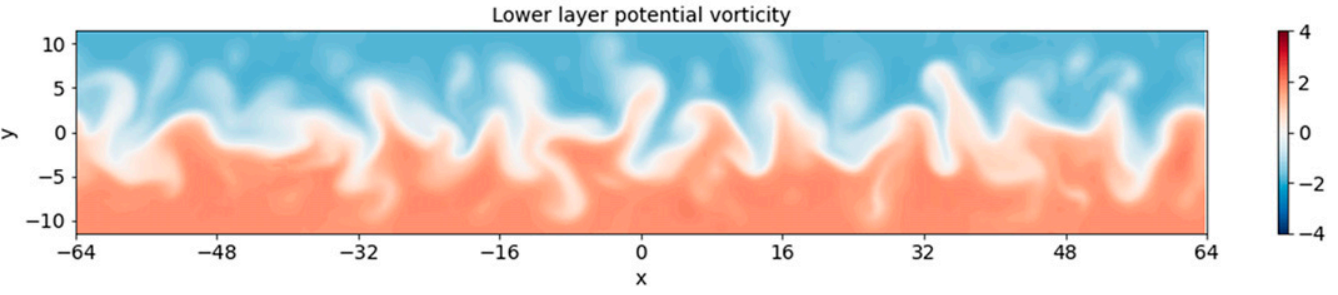

FIG. 1. Snapshots of potential vorticity for the (a),(b) momentum-converging and (c),(d) momentum-diverging solutions. The $\beta$ values are 0.2 and 0.02 , respectively. The color scales are consistent across the panels.

in the vertical integral, which is different from Chai et al. (2016) and Lutsko et al. (2017), who found EMF divergence in the lower layer but not in the vertical integral. The solutions in this regime have weak and easterly surface (i.e., lower-level) winds. Its eddy flux characteristics are compared with a more Earthlike, surface westerlies solution.

The parameters of the Earthlike control case are $\beta=0.2$, $r_{m}=0.2, r_{T}=0.03, \sigma=2$, and channel length $L=128$. For these parameters, the upper-level jet is reduced in strength from its equilibrium value in the absence of eddies by roughly a factor of 2 . The parameter values are adjusted from the control case of Lutsko et al. (2017), such that the surface jet is narrower and better defined when $\beta$ is decreased. The adjustment includes tripling of the damping rates $r_{m}$ and $r_{T}$, decrease of $\sigma$ from 3.5 to 2, and increase of $L$ from 46 to 128 . The use of a longer channel is important for solutions to converge in cases with smaller $\beta$, in which the EMF direction is sensitive to $L$, discussed in section 5 . With the standard $\beta=0.2$, our adjusted parameters generate solutions qualitatively similar to the synoptic variability in midlatitudes on Earth.

Figures 1a and 1b show snapshots of the total PV (which is $q+\beta y)$ in both layers in the statistically steady state of the
Earthlike control case. The solution captures Earthlike PV structures including waves, thin filaments, and isolated vortices in the upper layer. In the lower layer, the zonal-mean PV gradient as well as the perturbation are much weaker. Between the upper and lower layers, the zonal-mean PV gradients are reversed, satisfying the necessary condition for baroclinic instability.

Figures $1 \mathrm{c}$ and $1 \mathrm{~d}$ show snapshots of the total PV with $\beta=$ 0.02 , an order of magnitude smaller than the control case. The other parameters remain the same. Compared with the $\beta=0.2$ solution, isolated vortices become more common. A key qualitative difference is the longer dominant zonal wavelength, which will be discussed further in the following.

Figure 2 compares the zonal-mean zonal wind for the two $\beta$ values in Fig. 1. The upper-layer wind and vertical wind shear are maximized at $y=0$ in both solutions. The upper-layer wind is broader, and the surface wind is weaker in the small $\beta$ solution. Westerly surface winds occur around $y=0$ for $\beta=$ 0.2 , similar to Earth's atmosphere. It changes sign with latitude, as the conservation of momentum requires the domain integral of $r_{m} \bar{u}_{2}$ to be zero. On the other hand, easterly surface winds occur around $y=0$ for $\beta=0.02$. While very weak in this case, the surface easterlies can be larger when $\sigma$ is reduced as will be 

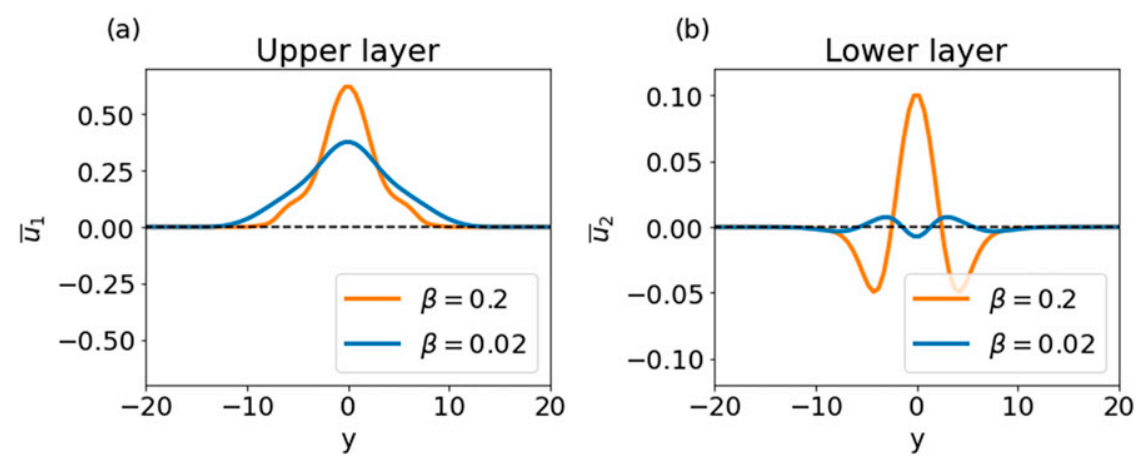

FIG. 2. The zonal-mean zonal winds in the (a) upper and (b) lower layers. The directions of lower-layer winds at the jet core are opposite between the $\beta=0.2$ and $\beta=0.02$ cases.

shown in section 5. Figure 3 compares the eddy momentum fluxes and eddy heat fluxes between the two solutions. As required by momentum conservation [i.e., Eq. (3)], the vertically integrated EMF converges toward the jet in the solution with surface westerlies, and it diverges from the jet in the solution with surface easterlies. The vertical integral is dominated by the upper layer in both cases.

The vertical wind shear and eddy heat flux both peak at the center of the baroclinic zone, indicating maximal baroclinic instability. Baroclinic instability is traditionally thought of as the source of waves, especially in the upper layer with its larger zonal-mean PV gradients. These waves transport momentum toward their source at the jet core, a direction of transport that can be understood from the Rossby wave group velocity, or, more generally, from the movement of pseudomomentum (i.e., wave activity) away from the jet. The divergence of momentum at the center of the baroclinic zone in the surface easterlies solution provides a counterexample in which this classical, quasi-linear picture is insufficient.

Figure 4 shows that the upper-layer EMF convergence decreases monotonically with decreasing $\beta$, and eventually changes sign. It is evaluated at the jet core and is normalized by the eddy heat flux, which is always positive. Because the vertically integrated EMF convergence is dominated by the upper layer, surface
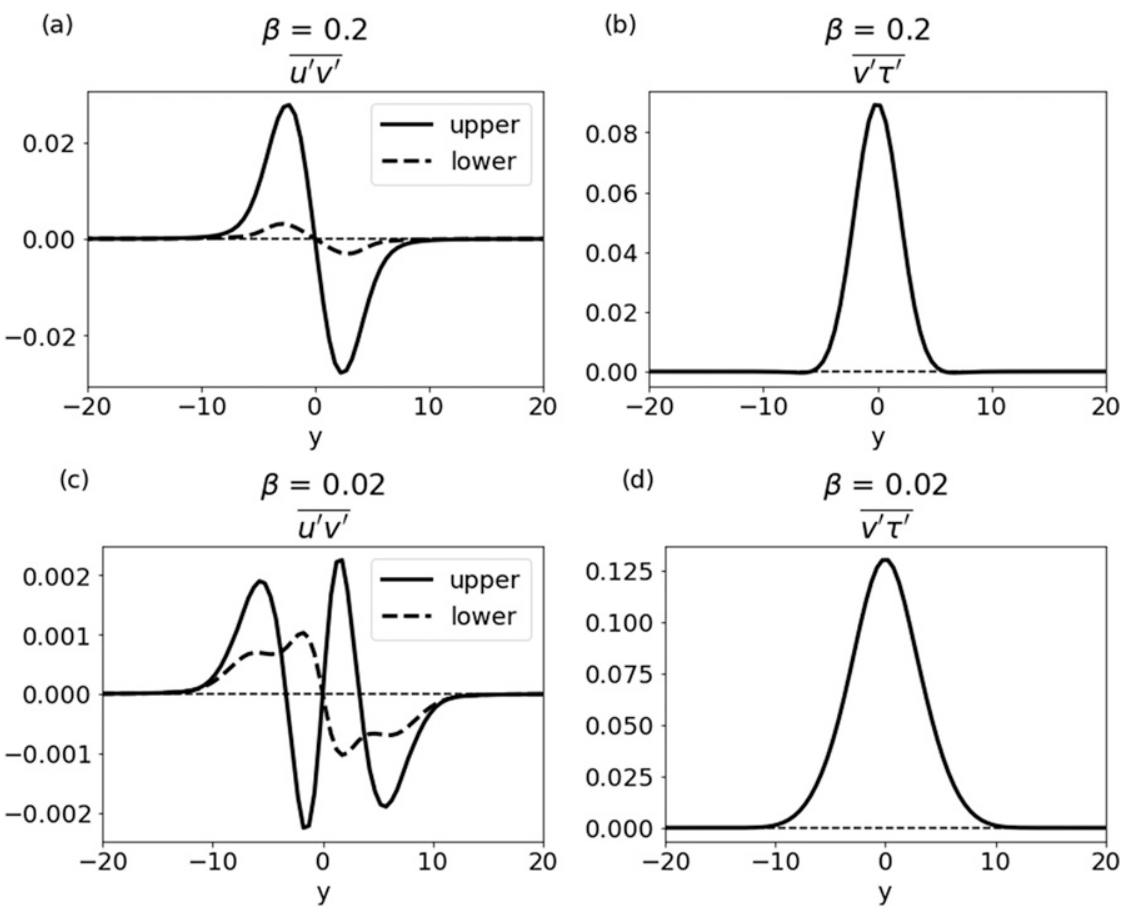

FIG. 3. The zonal-mean EMF and eddy heat flux for the (a),(b) $\beta=0.2$ and (c),(d) $\beta=0.02$ cases. The momentum flux convergence can have either sign at the jet core, where the heat flux and baroclinic instability is maximized. 


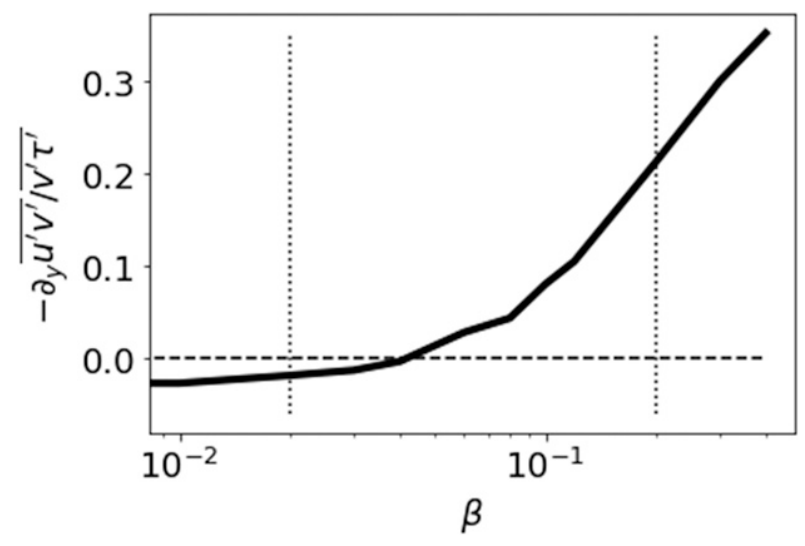

FIG. 4. The upper-layer EMF convergence normalized by the eddy heat flux at the jet core. The vertical dotted lines indicate the $\beta$ values of the experiments shown in Figs. 1-3. The classical theory of wave-mean flow interactions qualitatively describes the large $\beta$ limit but not the small $\beta$ limit.

easterlies are present in the small $\beta$ limit and surface westerlies in the large $\beta$ limit, with the transition near $\beta=0.04$.

The reversed EMF direction corresponds to a reversed direction of the barotropic conversion in the energy cycle. The barotropic conversion is $\overline{u^{\prime} v^{\prime}} \partial_{y} \bar{u}$, which represents conversion from the eddy kinetic energy (EKE) to the zonal kinetic energy (ZKE) when positive. When integrated over the domain, it can be expressed as the zonal wind multiplied by EMF convergence. Since the integral is dominated by its value at the jet core in the upper layer, the barotropic conversion has the same qualitative dependence on $\beta$ as the EMF convergence. In particular, in the momentum-diverging small $\beta$ limit, eddies on average remove energy as well as momentum from the jet.

Spectral analysis shows that the small ratio between EMF convergence and eddy heat flux is a result of cancellation between small and large wavenumbers. Figure 5a shows the EMF convergence decomposed by zonal wavenumbers $(k)$. It is evaluated at $y=0$ in the upper layer, which is much larger than in the lower layer for all wavenumbers in all experiments. The EMF convergence for each wavenumber is normalized by the total heat flux, such that the sum over all wavenumbers equals the total upper-layer EMF convergence divided by the total heat flux. The line style convention in this paper is that solutions with total upper-layer EMF convergence are represented by solid lines, and those with total upper-layer EMF divergence are represented by dashed lines.

For $\beta=0.2$, the spectrum peaks at the deformation wavenumber, indicating the importance of baroclinic instability. These wavenumbers converge momentum toward the jet, consistent with the most unstable waves in the classical quasilinear paradigm. As $\beta$ decreases, contributions from these momentum-converging large wavenumbers are reduced, as they transfer energy to small wavenumbers rather than the zonal flow. Contributions from smaller wavenumbers then become more important in setting the total EMF direction. In contrast to the most unstable wavenumbers, wavenumbers smaller than 0.5 diverge momentum regardless of $\beta$. The activation of these small wavenumbers reverses the direction of total EMF with sufficiently small $\beta$. Therefore, the $\beta$ dependence of total EMF direction can be understood as a competition between the momentum-converging large wavenumbers and momentum-diverging small wavenumbers. When $\beta$ is larger, the former dominates, leading to the momentum-converging (surface westerlies) regime. When $\beta$ is smaller, the opposite occurs, leading to the momentum-diverging (surface easterlies) regime. This regime transition cannot be explained by quasi-linear arguments that ignore nonlinear wave-wave interactions.

To demonstrate the importance of nonlinear wave-wave interactions in causing the gradual transition from the momentum-converging to momentum-diverging regime with (a)

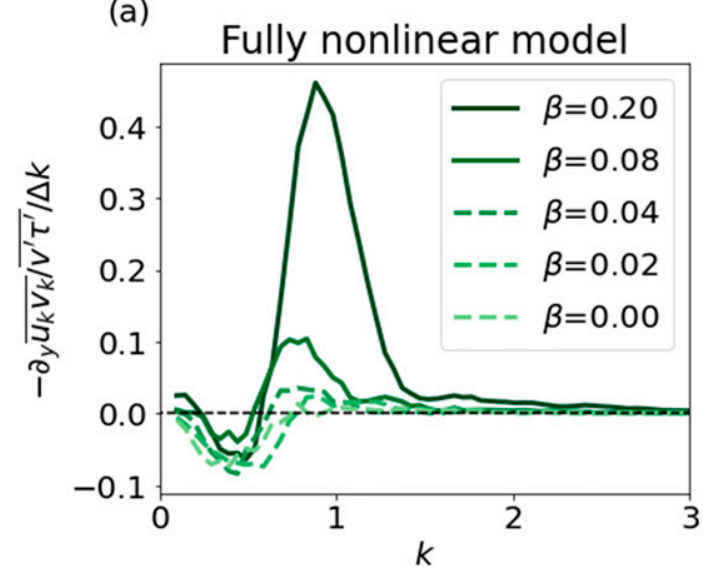

(b)

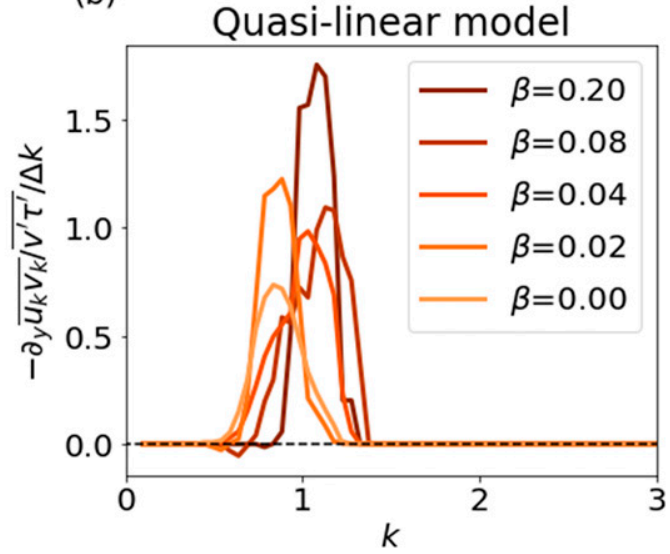

FIG. 5. Convergence of momentum by each zonal wavenumber $(k)$ at the jet core in the upper layer across a range of $\beta$ for (a) the fully nonlinear model and (b) the quasi-linear model. Solid curves represent convergence and dashed curves represent divergence when summed over all wavenumbers. The values are normalized by the eddy heat flux summed over all wavenumbers $\overline{v^{\prime} \tau^{\prime}}$. The characteristics of small wavenumbers are distinct between the fully nonlinear and quasi-linear models. 
decreasing $\beta$, we perform quasi-linear simulations and compare them with the fully nonlinear simulations. The quasi-linear solutions are obtained by running a separate set of experiments with modified equations. In the quasi-linear model, any product between two different wavenumbers is replaced with zero, but the product between the same wavenumbers is retained. Physically, this modification allows every wavenumber to interact with the zonal-mean flow but not with other wavenumbers directly. That is, decomposing the flows into the zonal-mean (denoted by the overbar) and the eddy (denoted by the prime) components, Eq. (1) is modified as

$$
\frac{\partial q_{1}}{\partial t}=-\overline{J\left(\psi_{1}^{\prime}, q_{1}^{\prime}\right)}-J\left(\bar{\psi}_{1}, q_{1}^{\prime}\right)-J\left(\psi_{1}^{\prime}, \bar{q}_{1}\right)-\beta v_{1}+r_{T} \frac{\left(\tau-\tau_{E}\right)}{\lambda^{2}}
$$

$$
\begin{aligned}
\frac{\partial q_{2}}{\partial t}= & -\overline{J\left(\psi_{2}^{\prime}, q_{2}^{\prime}\right)}-J\left(\bar{\psi}_{2}, q_{2}^{\prime}\right)-J\left(\psi_{2}^{\prime}, \bar{q}_{2}\right)-\beta v_{2} \\
& -r_{T} \frac{\left(\tau-\tau_{E}\right)}{\lambda^{2}}-r_{m} \nabla^{2} \psi_{2}
\end{aligned}
$$

where the eddy components of $J\left(\psi_{1}^{\prime}, q_{1}^{\prime}\right)$ and $J\left(\psi_{2}^{\prime}, q_{2}^{\prime}\right)$ are removed. The quasi-linear model can be thought of as intermediate between linear and fully nonlinear, isolating the wave-mean flow interaction from the nonlinear processes. It has served as a useful test bed for theoretical research and is easily implemented in pseudospectral models (e.g., O'Gorman and Schneider 2007). See Cehelsky and Tung (1991) for an example of quasi-linear simulations of this type in a two-layer QG model.

Figure $5 \mathrm{~b}$ shows the EMF convergence by each wavenumber normalized by the total heat flux for the quasi-linear solutions, using the same parameters as Fig. 5a. Comparison between the fully nonlinear and quasi-linear solutions indicates that the quasi-linear approximation becomes more inadequate as $\beta$ decreases. As described above, the removal of nonlinear wave-wave interactions prohibits energy transfer across wavenumbers directly, so the dominant wavenumbers remain close to 1 , which predominately converge momentum into the jet and transfer energy to the zonal flow. Even though the quasi-linear model does indicate a slight decrease of dominant wavenumbers with decreasing $\beta$ (cf. Cehelsky and Tung 1991), the smaller wavenumbers that diverge momentum are never active, implying that linear instability is insufficient in generating these wavenumbers. Due to the absence of the small wavenumbers, the momentumdiverging (surface easterlies) regime never occurs. The comparison suggests that wave-wave interactions are critical for generating the small wavenumbers and the associated EMF divergence.

\section{Mechanisms for the divergence of EMF}

In this section, we argue that the momentum-diverging small wavenumbers are generated by breaking of the most unstable wavenumbers near their critical latitudes. These latitudes are the pseudomomentum source for the small wavenumbers, where they converge momentum. The compensating divergence of momentum occurs at the center of the jet. An initial value experiment is set up to examine this process, followed by analyses of the phase speed-latitude spectra and the pseudomomentum budget in the statistically steady state of the fully nonlinear solutions to examine the behavior of different wavenumbers across latitudes.

To understand the nonlinear generation of small wavenumbers and its relation with the reversed EMF, we investigate an initial value problem perturbed around the time-mean, zonal-mean profiles of the fully nonlinear solutions. The linear damping terms are removed by setting $r_{m}=r_{T}=0$, similar to the configuration of Simmons and Hoskins (1978) for a multilayer baroclinic life cycle simulation. Initially, wavenumbers between 0.75 and 1.25 are perturbed. The range of perturbed wavenumbers is chosen such that all wavenumbers smaller than 0.5 would be excited in the model. An ensemble of 50 realizations with randomized initial amplitudes are performed and averaged over.

Figure 6 shows the evolution of kinetic energy and EMF convergence for the large and small wavenumbers for both $\beta$ values. Initially, a wave packet composed of the unstable wavenumbers grows exponentially. The wave packet develops from the center of the jet, where the vertical wind shear is maximized, and converges momentum toward the jet. Around $t=15$ for $\beta=0.2$ and $t=25$ for $\beta=0.02$, wave breaking occurs on the flanks of the jet. Around the same time and latitudes, small wavenumbers start to develop and converge momentum. The difference between the two $\beta$ values is that the small wavenumbers are much weaker than the large wavenumbers for $\beta=0.2$; while they have comparable magnitudes for $\beta=0.02$. The unforced initial value problem suggests that for both $\beta$, the breaking of unstable wavenumbers on the jet flanks generates smaller wavenumbers. They converge momentum on the flanks in both cases and diverge momentum at the jet core with small $\beta$.

To investigate the dynamics around the critical latitudes in the statistically steady state of the fully nonlinear solutions, Fig. 7 shows the phase speed-latitude spectra (Randel and Held 1991) of EMF convergence, along with the zonal-mean wind. It is generated by a similar procedure to Fig. 5, with an additional Fourier transform performed along $t$. In Fig. 7, the spectra are evaluated at a single zonal wavenumber $k$, and the $\omega$ axis is replaced with the zonal phase speed $\omega / k$. The upperlayer EMF convergence is normalized by the total heat flux at the jet core.

Figure 7a shows results for the deformation wavenumber with $\beta=0.2$, which is the peak of the EMF convergence spectrum in Fig. 5. The signal is concentrated within a narrow range of phase speeds, or equivalently a narrow range of frequencies given a constant wavenumber. This frequency range matches well the Rossby dispersion relation with meridional wavenumber 1 (not shown).

The meridional distribution of EMF convergence at this wavenumber is consistent with the classical quasi-linear paradigm. This wave is generated through baroclinic instability at the jet core, where the momentum is converged. It propagates away from the jet until approaching the critical latitudes, where the phase speed equals the zonal-mean wind speed. Rossby wave critical layer theory suggests wave breaking at those latitudes, where the momentum flux diverges. 


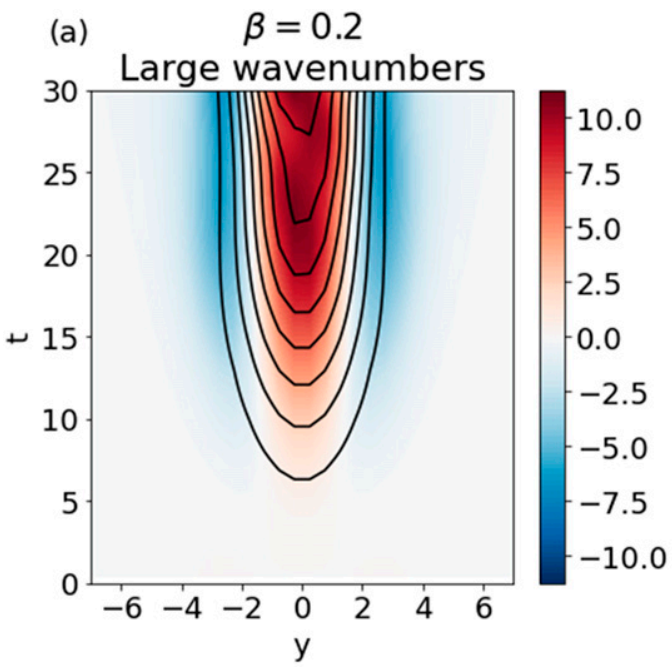

(b)

$\quad \beta=0.2$
Small wavenumbers

(c) $\quad \beta=0.02$

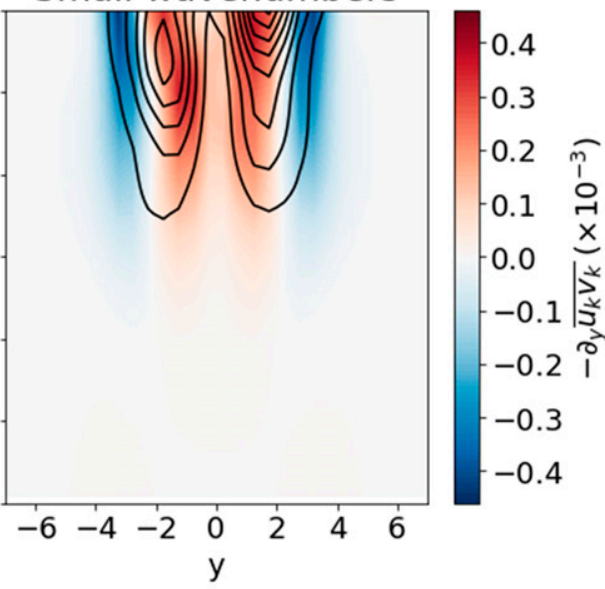

Large wavenumbers

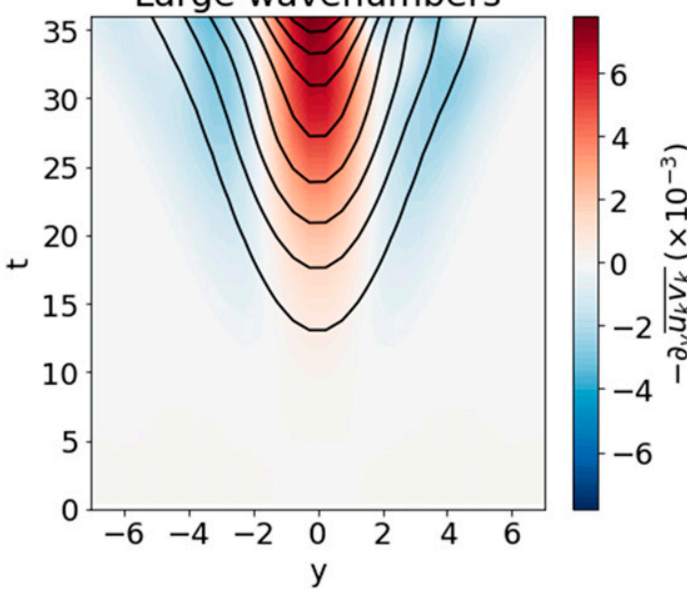

(d)

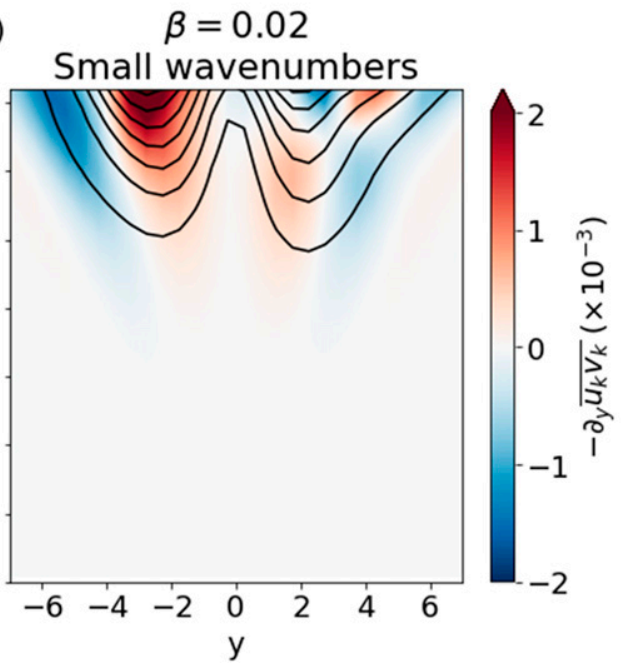

FIG. 6. Evolution of the upper-layer EKE (contour lines) and EMF convergence (color shading) as functions of time and latitude for (a) $0.75<k<1.25$ and (b) $k<0.5$, where $k$ is the zonal wavenumber. The mean profile is taken from a fully nonlinear simulation with $\beta=0.2$. (c),(d) As in (a) and (b), but with $\beta=0.02$.

On the other hand, Fig. $7 \mathrm{~b}$ shows a representative small wavenumber near the negative peak of the EMF convergence spectrum in Fig. 5. In contrast to the previous case, this wavenumber is forced over a broad range of phase speeds, rather than the phase speed of the breaking wave. The momentum flux is convergent near the critical latitudes of the breaking wave at the deformation wavenumber, and the compensating momentum flux divergence occurs at the jet core. Although this is seen for a range of phase speeds, westward phase speeds are dominant.

Aggregated over all wavenumbers, Fig. 8a shows that the latitude of EMF divergence follows the critical latitude for the eastward-propagating fast and large wavenumbers. The slow and small wavenumbers converge momentum around the same latitudes, and they diverge momentum at the jet core. Figure $8 \mathrm{~b}$ shows the same plot for the case with $\beta=0.02$. The fast eastward wavenumbers that converge momentum toward the jet resemble those in the $\beta=0.2$ case. They diverge momentum near the critical latitudes, but the signal is much weaker. The total EMF is dominated by the momentum-diverging small wavenumbers with more westward phase speeds.

To provide further evidence for the nonlinear generation of small wavenumbers on the jet flanks, Fig. 9 shows the pseudomomentum budget in the statistically steady state of the fully nonlinear solutions. The zonal-mean pseudomomentum equation for each zonal wavenumber $k$ in the upper layer is

$$
\frac{\partial}{\partial t} \frac{\overline{q_{k}^{2}}}{\gamma}=-\overline{v_{k} q_{k}}-\frac{\overline{q_{k} J(\psi, q)_{k}}}{\gamma}+r_{T} \frac{\overline{q_{k} \tau_{k}}}{\gamma}
$$

where $\gamma=\beta+\partial_{y} \bar{q}_{1}$ is the zonal-mean PV gradient. Under the assumption that $\gamma$ is constant in time, the left-land side is zero in the time mean. Expanding the PV flux into the vorticity flux and the heat flux, Eq. (6) is rewritten as

$$
\overline{v_{k} \zeta_{k}} \approx \overline{v_{k} \tau_{k}}-\frac{\overline{q_{k} J(\psi, q)_{k}}}{\gamma}+r_{T} \frac{\overline{q_{k} \tau_{k}}}{\gamma} .
$$



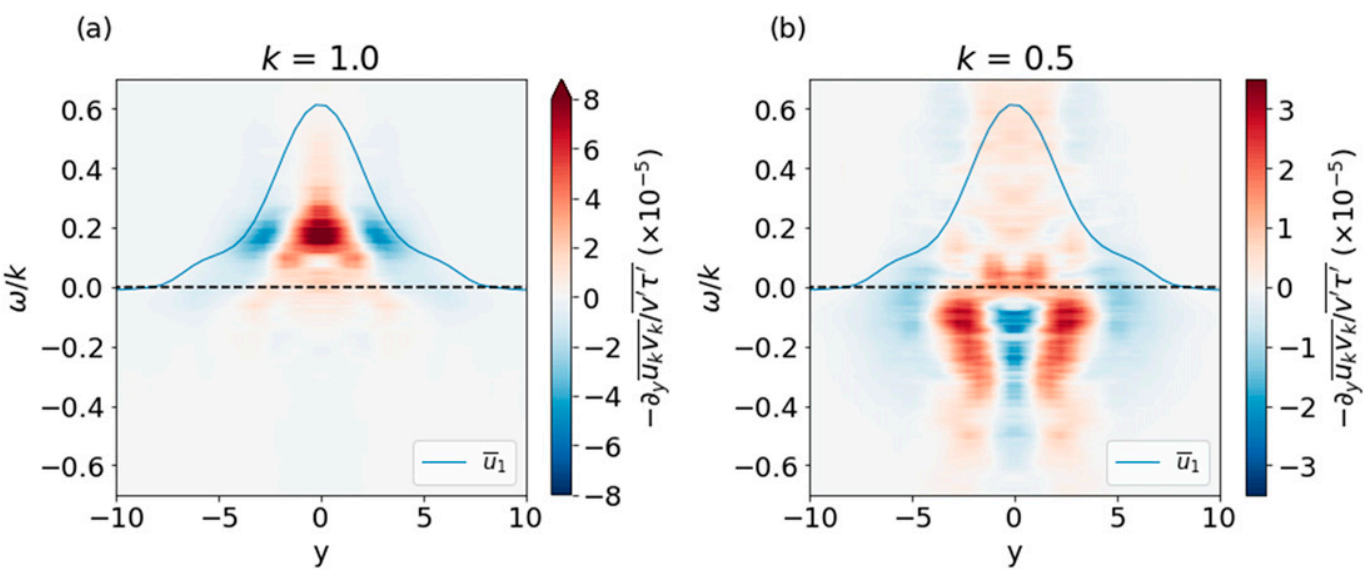

FIG. 7. The phase speed-latitude spectra of upper-layer EMF convergence for the fully nonlinear, $\beta=0.2$ solution. (a) The most unstable wavenumber that converges momentum at the jet core and diverges momentum close to the critical latitudes where $\omega / k \approx \bar{u}_{1}$ (solid blue line). (b) A representative small wavenumber that diverges momentum at the jet core. The values have been normalized by the total heat flux.

The first term on the right-hand side, which is the heat flux, is the linear source of pseudomomentum. The second term, which is a third-order statistic, is the nonlinear source due to wave-wave interactions. This term is not present in the quasilinear model. The third term is dissipation due to thermal damping.

The terms in Eq. (7) are diagnosed in Fig. 9 for the wavenumber that causes the most EMF divergence at the jet core for each $\beta$. As in the phase speed-latitude analysis, the small wavenumbers converge EMF (i.e., positive vorticity flux) on the jet flanks, and these latitudes correspond to the peaks of the nonlinear pseudomomentum source. The heat flux term cannot explain the generation of small wavenumbers on the jet flanks. Due to the temporal variability, there is a small residual in the diagnostics indicated by the dashed curve in Fig. 9, which has little latitudinal structure and does not influence the correspondence of peaks of EMF convergence and nonlinear pseudomomentum source on the jet flanks.
The physical picture emerging from Figs. 6-9 is the following. Consistent across $\beta$, the small wavenumbers $(k \lesssim 0.5)$ are generated by breaking of large wavenumbers $(k \approx 1)$ near their critical latitudes. Around these latitudes, the small wavenumbers converge EMF as their pseudomomentum is generated, mainly by nonlinear wave-wave interactions. The small-wavenumber EMF diverges at the jet core due to the conservation of momentum. The $\beta$ parameter determines the relative contributions of small and large wavenumbers to the total EMF.

\section{Dependence of EMF on the surface drag, width of baroclinic zone, and channel length}

The fact that smaller wavenumbers tend to diverge momentum holds in a larger parameter space, across a range of surface drag $r_{m}$, meridional scale of thermal forcing $\sigma$, and channel length $L$ investigated in this section. Previous sections suggest (a)

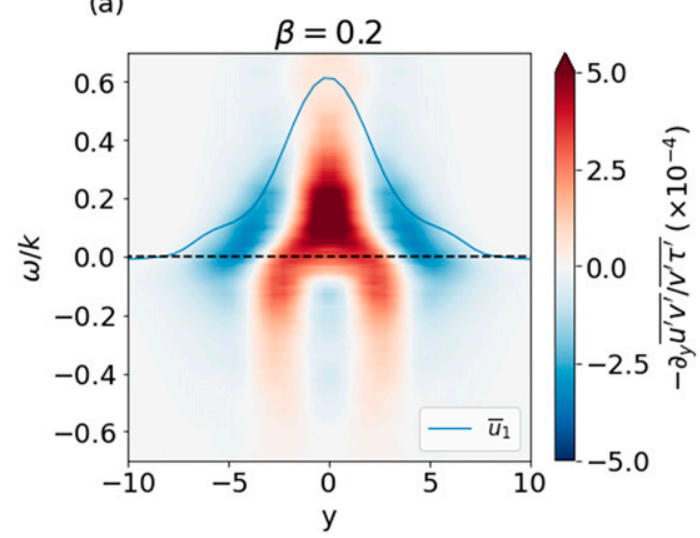

(b)

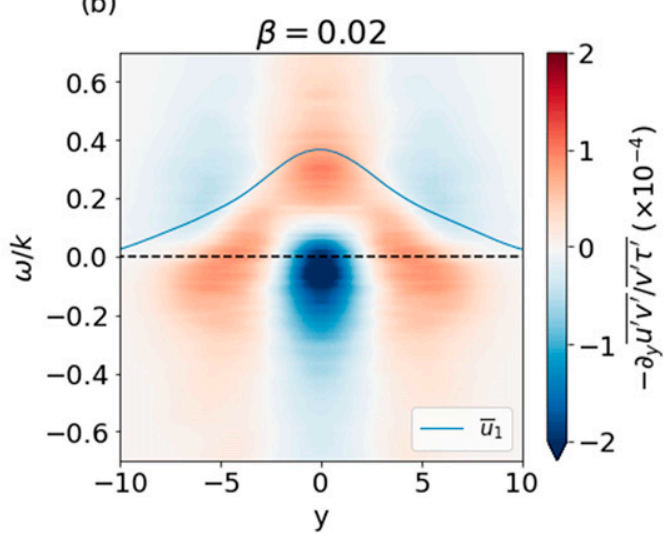

FIG. 8. The phase speed-latitude spectra of upper-layer momentum flux convergence aggregated over all wavenumbers for (a) $\beta=0.2$ and (b) $\beta=0.02$. The values have been normalized by their respective total heat fluxes, which are the same as in Fig. 7. 

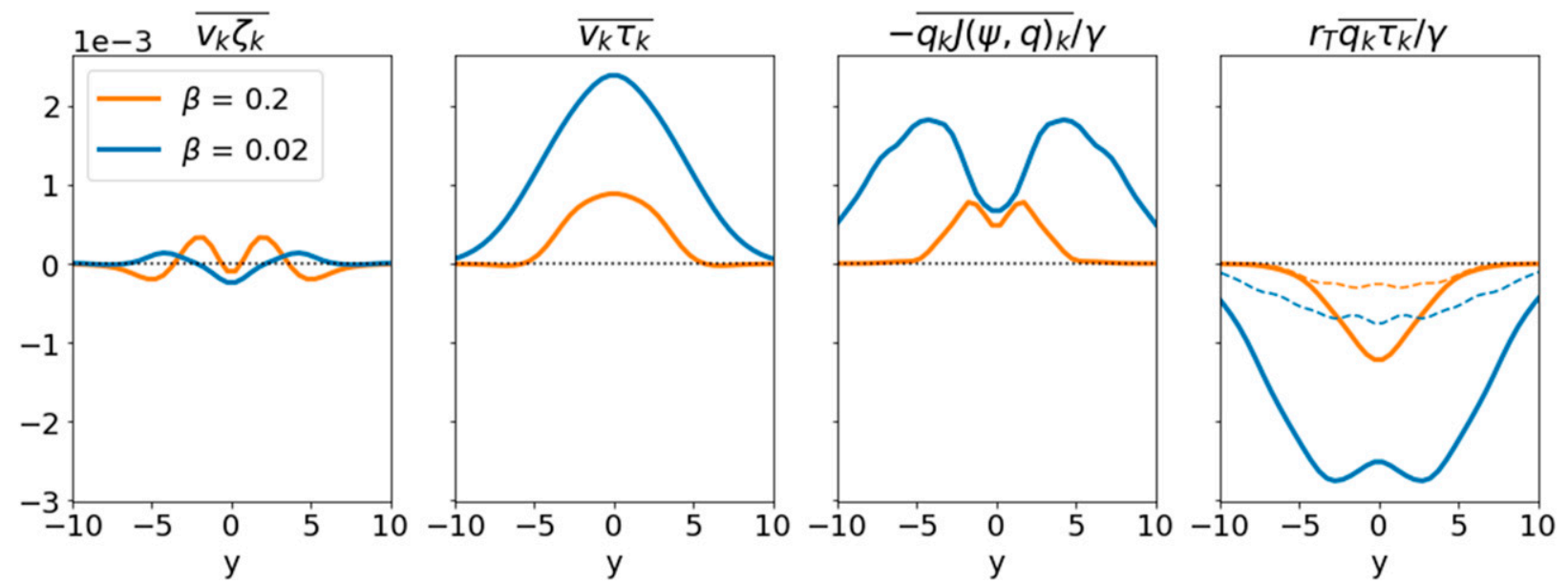

FIG. 9. The upper-layer pseudomomentum budget of wavenumbers that diverge the most momentum from the jet, for each term in Eq. (6). The residual in the time mean is represented by the dashed curve. The wavenumbers displayed are $k=0.5$ for $\beta=0.2$ and $k=0.25$ for $\beta=0.02$. The budget suggests that the EMF convergence (equivalent to the vorticity flux) on the jet flanks corresponds to the nonlinear source of pseudomomentum due to wave-wave interactions.

that the regime transition from EMF convergence to divergence is caused by a competition between the momentumdiverging small wavenumbers and the momentum-converging large wavenumbers. As $\beta$ decreases, relatively more small wavenumbers are generated by breaking of large wavenumbers. Besides decreasing $\beta$, decreasing the frictional strength is known to halt the upscale energy transfer (e.g., Thompson and Young 2007), which we confirm in the following as another path that leads to the EMF divergence regime. In addition, the width of thermal forcing and the length of the zonal channel are perturbed to alter the relative strengths of small and large wavenumbers.

Figures 10a and 10b show the spectra of EMF convergence, similar to Fig. 5a except with varying the mechanical damping $r_{m}$ and fixed $\beta$. Recall that the control $r_{m}=0.2$, with which $\beta=0.2$ gives EMF convergence and $\beta=0.02$ gives EMF divergence at the center of the jet. Regardless of decreasing $\beta$ or decreasing $r_{m}$, the qualitative changes are the same: large wavenumbers converge momentum and small wavenumbers diverge momentum. With sufficiently small $\beta$ and $r_{m}$, the small wavenumbers become dominant, and the total vorticity flux direction is reversed.

Figure 10c shows how the ratio between the upper-layer EMF convergence and heat flux depends jointly on $r_{m}$ and $\beta$. Because the dimensional $\beta$ and drag coefficient have different units, the axes are converted to wavenumber scales by a simple combination with the velocity scale of the thermal equilibrium wind shear. That is, in the dimensional form, the wavenumber scales are $r_{m} / U$ and $\sqrt{\beta / U}$ respectively. Given our nondimensionalization, $U$ is set to unity in these expressions. Figure 10c shows that these two wavenumber scales play similar roles in determining the EMF direction: it is convergent (a)

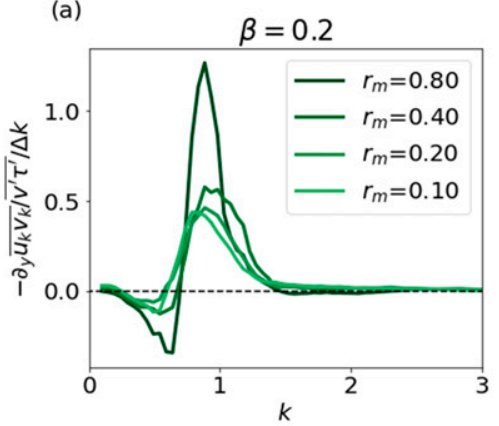

(b)

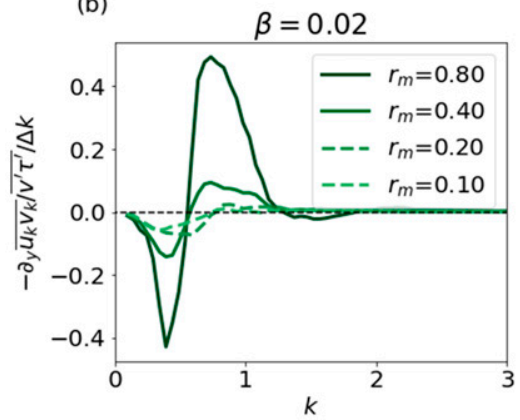

(c)

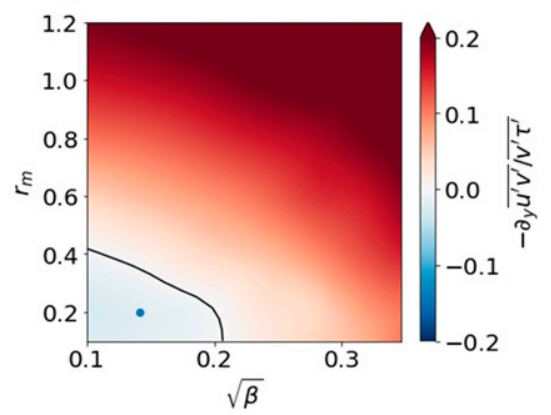

FIG. 10. Spectra of upper-layer EMF convergence normalized by the total heat flux, as in Fig. 5 except for varying $r_{m}$ with (a) $\beta=0.2$ and (b) $\beta=0.02$. (c) Ratio between the total upper-layer EMF convergence and heat flux for solutions with a range of $r_{m}$ and $\beta$. The black contour indicates zero EMF convergence. The blue circle indicates parameter values for the standard $\beta=0.02$ experiment. The standard $\beta=0.2$ experiment is outside the range shown here, as it is chosen to focus on the regime transition. The axes are converted to wavenumber scales by combining with the thermal equilibrium wind shear. EMF divergence solutions tend to occur with weak surface drag and small $\beta$. 

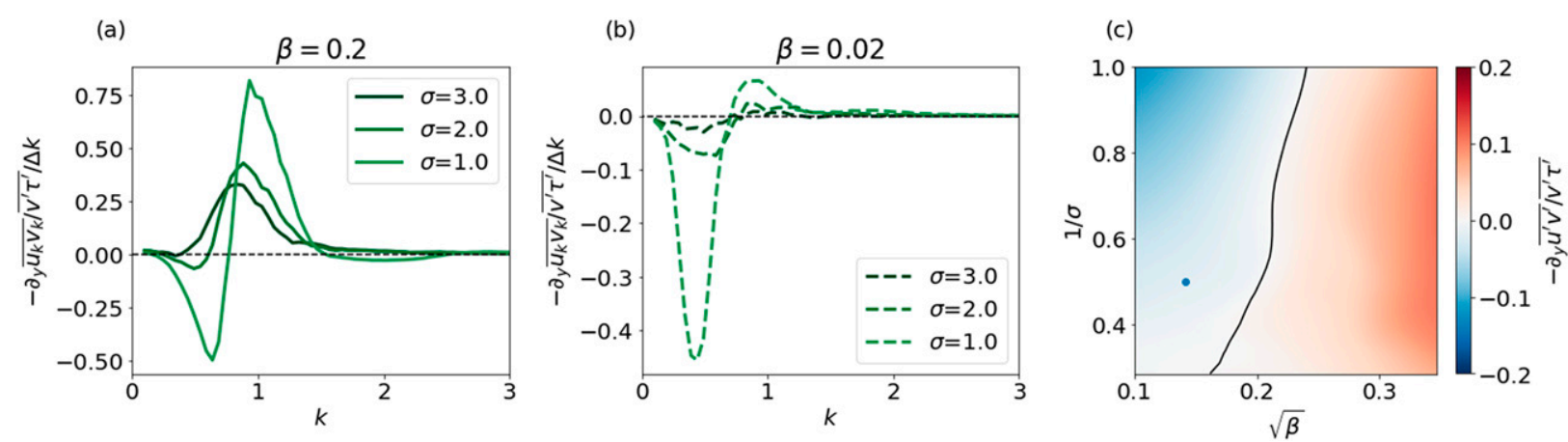

FIG. 11. As in Fig. 10, but for varying $\sigma$ with (a) $\beta=0.2$ and (b) $\beta=0.02$. (c) Ratio between the total EMF convergence and heat flux as functions of wavenumber scales $1 / \sigma$ and $\sqrt{\beta}$. EMF divergence solutions tend to occur with narrow jets and small $\beta$.

when the wavenumber scales are large, and it is divergent when the wavenumber scales are small.

The similar effects of $\beta$ and $r_{m}$ are likely related to the halting of upscale energy transfer. The halting wavenumber has been found to increase with $\beta$ and $r_{m}$ in numerical simulations of two-layer QG turbulence (e.g., Thompson and Young 2007). The best way of thinking about the halting scales in the two-layer context remains a topic of current research (Chang and Held 2019; Gallet and Ferrari 2020), but nevertheless the simplest barotropic halting scales appear to be useful here.

Figure 11 is similar to Fig. 10 except with varying $\sigma$, the meridional scale of thermal forcing, which sets the width of the radiative-equilibrium jet. Over the parameter range examined, the PV gradients never change sign in the horizontal direction, either in the equilibrated jets or in the forced jets in the absence of eddies. For either $\beta=0.2$ or 0.02 , the momentum-diverging small wavenumbers are more active with smaller $\sigma$. The solution becomes homogeneous in the limit of infinite $\sigma$, in which the EMF convergence is zero.

The joint dependence of upper-layer EMF convergence with $\sigma$ and $\beta$ is shown in Fig. 11c. Similar to Fig. 10c, the ratio between EMF convergence and heat flux is plotted as a function of wavenumber scales $1 / \sigma$ and $\sqrt{\beta}$. The EMF direction is relatively insensitive to $\sigma$ for larger $\beta$, and it transitions from positive to negative with decreasing $\sigma$ for smaller $\beta$. The enhanced small wavenumbers transport momentum out of the jet core, which counters the sharpening effect by thermal forcing on the jet due to decreasing $\sigma$.

Finally, the zonal length of the channel, $L$, directly controls the smallest resolvable wavenumber. For $\beta=0.2$ (Fig. 12a), the EMF spectra have qualitatively converged with $L$ : the EMF convergence is dominated by wavenumber 1 . On the other hand, the spectra are qualitatively different across $L$ for $\beta=$ 0.02 (Fig. 12b). The small wavenumbers are dominant when they are allowed in long channels and result in net EMF divergence. When they are eliminated in the $L=16$ short channel, the net EMF converges toward the jet.

In Earthlike settings, the channel length is often set approximately to 6 times the most unstable wavelength (e.g., Phillips 1956). For our Earthlike solutions with $\beta=0.2$, increasing the channel length beyond this value would not alter the mean climate qualitatively. However, when the parameters are perturbed such that smaller wavenumbers are active, for instance with $\beta=0.02$, the channel length may change the mean climate, including the EMF and surface wind directions.

\section{Conclusions}

This paper describes nonlinear mechanisms that determine the direction of the eddy momentum flux (EMF) in a forceddissipative two-layer quasigeostrophic model. Classical theories for the EMF often involve the quasi-linear assumption and emphasize the wave-mean flow interactions (e.g., Held 1975), whereas the roles of wave-wave interactions have been largely unexplored. In this study, we show that the classical quasilinear picture is insufficient for understanding the EMF direction in regimes with small $\beta$, small surface drag, narrow width of the baroclinic zone, or large zonal channel length.

As $\beta$ decreases, a flow regime with upper-layer EMF divergence from the jet emerges, despite having otherwise similar qualitative features to the Earthlike flows. Spectral analysis shows that the sign of EMF convergence is opposite between the most unstable and the smaller wavenumbers. The classical theories of wave-mean flow interactions explain the EMF convergence toward the jet at the most unstable wavenumbers. However, a range of smaller wavenumbers is found to diverge EMF, which results in a net EMF divergence when the $\beta$ parameter is small. Comparison between fully nonlinear and quasi-linear simulations indicates that the smaller wavenumbers do not exist without wave-wave interactions.

The most unstable wavenumbers and the smaller wavenumbers are generated at different latitudes, resulting in different EMF directions. Initial value experiments in the fully nonlinear model suggest that breaking of the most unstable wavenumbers generates smaller wavenumbers. In the statistically steady state, the most unstable wavenumbers diverge momentum near their critical latitudes, indicated by the phase speed-latitude spectra. Around these latitudes, the small wavenumbers converge momentum as the pseudomomentum is generated by the nonlinear wave-wave interaction term. The compensating EMF divergence for small wavenumbers occurs at the jet core. The dynamics are summarized in Fig. 13.

While the analysis and numerical results are kept in a fairly idealized context in this paper, we believe that our findings may 
(a)

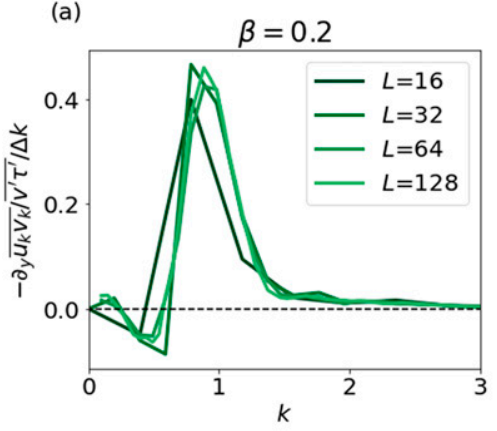

(b)

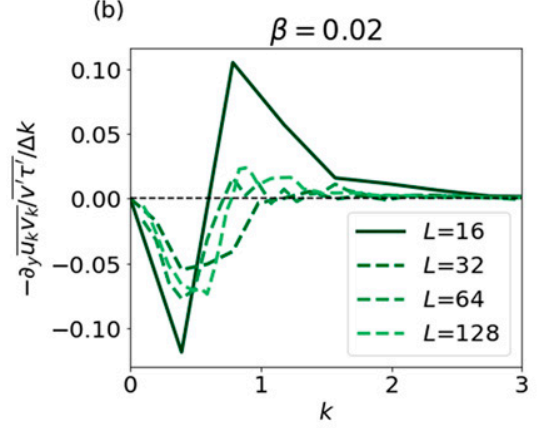

(c)

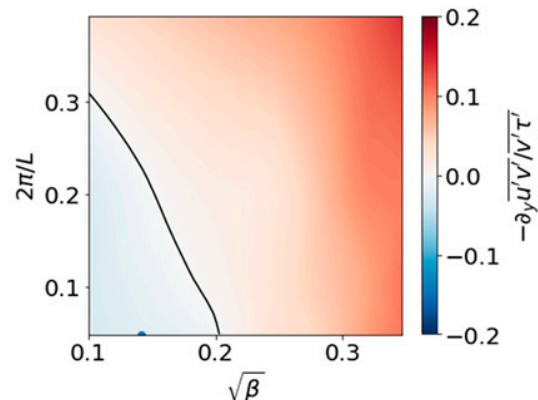

FIG. 12. As in Fig. 10, but for varying channel length $L$ with (a) $\beta=0.2$ and (b) $\beta=0.02$. (c) Ratio between the total EMF convergence and heat flux as functions of wavenumber scales $2 \pi / L$ and $\sqrt{\beta}$. EMF divergence solutions tend to occur with long channel lengths and small $\beta$.

be relevant in more realistic settings. In particular, the nonlinear generation of long waves by breaking unstable waves in our model is not restricted to small- $\beta$ regimes but is also found with Earthlike parameters. This is consistent with the observational study of Birner et al. (2013), who found that the downgradient PV flux associated with synoptic wave breaking is followed by an upgradient PV flux (equivalent to a pseudomomentum source) for the planetary waves on Earth. Because the breaking and nonlinear generation are not collocated spatially, this gives rise to a locally upgradient PV flux on the poleward side of the subtropical jet in the time mean. This is in some contrast with our two-layer results, for which only the longwave vorticity flux becomes upgradient on the jet flanks due to the much stronger baroclinic PV flux over the region. This difference is likely due to the idealizations in our simple model, especially the constant deformation radius and extreme vertical truncation, both of which may overemphasize the coupling between upper and lower levels as compared to the decoupling on the subtropical side of the storm track. ZuritaGotor and Vallis (2013) also found upgradient PV fluxes in an idealized primitive equation model with fine vertical structure and noted that this was possible due to the smallness of the baroclinic PV flux over the jet region, which they attributed to mixing barrier effects.

We highlight the implication of our results for the maintenance of midlatitude EMF convergence and surface westerlies. We show the limitation of the classical quasi-linear paradigm in explaining the emergence of upper-layer EMF divergence and surface easterlies in parameter regimes that allows significant wave-wave interactions. This in turn emphasizes the importance of the combination of Earthlike parameters, including the modest planetary radius as well as the coincidence of the Rhines scale and the Rossby radius of deformation, in limiting the inverse energy cascade in the atmosphere, which justifies
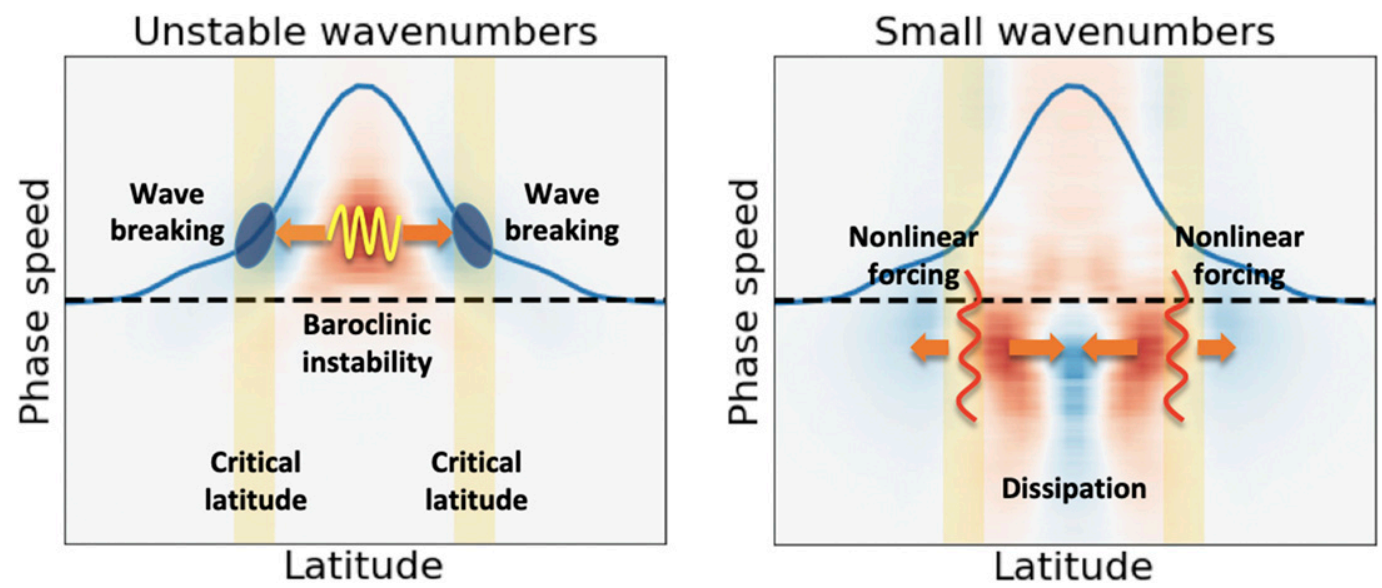

FIG. 13. A schematic diagram summarizing the dynamics of the most unstable wavenumbers and the small wavenumbers. The most unstable wavenumbers are generated by baroclinic instability at the jet core and break near the critical latitudes, where the zonal phase speed equals the mean zonal wind speed. Near the latitudes of wave breaking, the small wavenumbers are forced over a range of phase speeds and converge momentum (red shading). The compensating momentum flux divergence (blue shading) occurs at the jet core as the small wavenumbers dissipate. The blue curve represents the mean zonal wind speed in the upper layer. The arrows represent the direction of the pseudomomentum flux, which is opposite to the momentum flux. 
the qualitative adequacy of quasi-linear models in capturing the Earthlike climate.

Acknowledgments. We thank Stephen Garner, Olivier Pauluis, and Nicholas Lutsko for feedback on earlier versions of this manuscript. This report was prepared by TLH under Award NA14OAR4320106 from the National Oceanic and Atmospheric Administration, U.S. Department of Commerce. The statements, findings, conclusions, and recommendations are those of the author(s) and do not necessarily reflect the views of the National Oceanic and Atmospheric Administration, or the U.S. Department of Commerce. IMH and PZG are supported by the National Science Foundation Grant AGS-1733818. Data used in this research are archived on the Princeton University Research Computing Systems and are available upon request.

\section{REFERENCES}

Abernathey, R., and Coauthors, 2019: pyqg/pyqg: v0.3.0. Zenodo, https://doi.org/10.5281/zenodo.3551326.

Birner, T., D. W. J. Thompson, and T. G. Shepherd, 2013: Upgradient eddy fluxes of potential vorticity near the subtropical jet. Geophys. Res. Lett., 40, 5988-5993, https://doi.org/10.1002/ 2013 GL057728.

Cehelsky, P., and K. K. Tung, 1991: Nonlinear baroclinic adjustment. J. Atmos. Sci., 48, 1930-1947, https://doi.org/10.1175/ 1520-0469(1991)048<1930:NBA>2.0.CO;2.

Chai, J., M. Jansen, and G. K. Vallis, 2016: Equilibration of a baroclinic planetary atmosphere toward the limit of vanishing bottom friction. J. Atmos. Sci., 73, 3249-3272, https://doi.org/ 10.1175/JAS-D-15-0329.1.

Chang, C.-Y., and I. M. Held, 2019: The control of surface friction on the scales of baroclinic eddies in a homogeneous quasigeostrophic two-layer model. J. Atmos. Sci., 76, 1627-1643, https://doi.org/10.1175/JAS-D-18-0333.1.

Farrell, B. F., and P. J. Ioannou, 2003: Structural stability of turbulent jets. J. Atmos. Sci., 60, 2101-2118, https://doi.org/ 10.1175/1520-0469(2003)060<2101:SSOTJ $>2.0$. CO 2 .

Feldstein, S. B., and I. M. Held, 1989: Barotropic decay of baroclinic waves in a two-layer beta-plane model. J. Atmos. Sci., 46, 3416-3430, https://doi.org/10.1175/1520-0469(1989)046<3416: BDOBWI $>2.0 . \mathrm{CO} ; 2$.

Gallet, B., and R. Ferrari, 2020: The vortex gas scaling regime of baroclinic turbulence. Proc. Natl. Acad. Sci. USA, 117, 44914497, https://doi.org/10.1073/pnas.1916272117.

Held, I. M., 1975: Momentum transport by quasi-geostrophic eddies. J. Atmos. Sci., 32, 1494-1497, https://doi.org/10.1175/ 1520-0469(1975)032<1494:MTBQGE>2.0.CO;2.

Kuo, H., 1951: Vorticity transfer as related to the development of the general circulation. J. Meteor., 8, 307-315, https://doi.org/ 10.1175/1520-0469(1951)008<0307:VTARTT>2.0.CO;2.

Lee, S., 1997: Maintenance of multiple jets in a baroclinic flow. J. Atmos. Sci., 54, 1726-1738, https://doi.org/10.1175/15200469(1997)054<1726:MOMJIA>2.0.CO;2.
— observations. J. Atmos. Sci., 50, 1413-1428, https://doi.org/ 10.1175/1520-0469(1993)050<1413:BWPIMA>2.0.CO;2.

Lutsko, N. J., I. M. Held, and P. Zurita-Gotor, 2015: Applying the fluctuation-dissipation theorem to a two-layer model of quasigeostrophic turbulence. J. Atmos. Sci., 72, 3161-3177, https:// doi.org/10.1175/JAS-D-14-0356.1.

,,--- , $\longrightarrow$, and A. K. O'Rourke, 2017: Lower-tropospheric eddy momentum fluxes in idealized models and reanalysis data. J. Atmos. Sci., 74, 3787-3797, https://doi.org/10.1175/ JAS-D-17-0099.1.

O'Gorman, P. A., and T. Schneider, 2007: Recovery of atmospheric flow statistics in a general circulation model without nonlinear eddy-eddy interactions. Geophys. Res. Lett., 34, L22801, https://doi.org/10.1029/2007GL031779.

O'Rourke, A. K., and G. K. Vallis, 2016: Meridional Rossby wave generation and propagation in the maintenance of the wintertime tropospheric double jet. J. Atmos. Sci., 73, 2179-2201, https://doi.org/10.1175/JAS-D-15-0197.1.

Pavan, V., and I. M. Held, 1996: The diffusive approximation for eddy fluxes in baroclinically unstable jets. J. Atmos. Sci., 53, 1262-1272, https://doi.org/10.1175/1520-0469(1996)053<1262: TDAFEF $>2.0 . \mathrm{CO} ; 2$.

Phillips, N. A., 1956: The general circulation of the atmosphere: A numerical experiment. Quart. J. Roy. Meteor. Soc., 82, 123-164, https://doi.org/10.1002/qj.49708235202.

Randel, W. J., and I. M. Held, 1991: Phase speed spectra of transient eddy fluxes and critical layer absorption. J. Atmos. Sci., 48, 688-697, https://doi.org/10.1175/1520-0469(1991) $048<0688$ :PSSOTE $>2.0$. CO $; 2$.

Simmons, A. J., and B. J. Hoskins, 1978: The life cycles of some nonlinear baroclinic waves. J. Atmos. Sci., 35, 414-432, https:// doi.org/10.1175/1520-0469(1978)035<0414:TLCOSN>2.0.CO;2.

Thompson, A. F., and W. R. Young, 2007: Two-layer baroclinic eddy heat fluxes: Zonal flows and energy balance. J. Atmos. Sci., 64, 3214-3231, https://doi.org/10.1175/JAS4000.1.

Vallis, G. K., 2017: Atmospheric and Oceanic Fluid Dynamics: Fundamentals and Large-Scale Circulation. 2nd ed. Cambridge University Press, 946 pp.

Wang, L., M. Jansen, and R. Abernathey, 2016: Eddy phase speeds in a two-layer model of quasigeostrophic baroclinic turbulence with applications to ocean observations. J. Phys. Oceanogr., 46, 1963-1985, https://doi.org/10.1175/JPO-D-15-0192.1.

Wiin-Nielsen, A., and J. Sela, 1971: On the transport of quasi-geostrophic potential vorticity. Mon. Wea. Rev., 99, 447-459, https://doi.org/ 10.1175/1520-0493(1971)099<0447:OTTOQP>2.3.CO;2.

Zhang, W., C. L. P. Wolfe, and R. Abernathey, 2020: Role of surface-layer coherent eddies in potential vorticity transport in quasigeostrophic turbulence driven by eastward shear. Fluids, 5, 2, https://doi.org/10.3390/fluids5010002.

Zurita-Gotor, P., and R. S. Lindzen, 2006: A generalized momentum framework for looking at baroclinic circulations. J. Atmos. Sci., 63, 2036-2055, https://doi.org/10.1175/JAS3737.1.

— popause height in an idealized gray radiation model. J. Atmos. Sci., 70, 2272-2292, https://doi.org/10.1175/JAS-D-12-0209.1. 